\title{
Abgehängt und doch freiwillig engagiert? Ein Projekt zur Erreichung und Unterstützung benachtei- ligter junger Menschen im Freiwilligendienst
}

\author{
Maike Herrmann \\ Dipl.-Päd. \\ Bundesarbeitsgemeinschaft Evangelische Jugendsozialarbeit e. V. \\ Projektleitung Freiwilligendienste|m.herrmann@bagejsa.de
}

Junge Menschen mit niedrigen Bildungsabschlüssen oder sozial Benachteiligte sind in den Jugendfreiwilligendiensten wie dem Freiwilligen Sozialen Jahr immer noch unterrepräsentiert. Jugendliche aus dem Bereich Jugendsozialarbeit, die oftmals besonders benachteiligt sind, finden bisher nur selten Zugang zu Freiwilligendiensten, da häufig geeignete Strukturen und Formate für diese Zielgruppe fehlen. Die Bundesarbeitsgemeinschaft Evangelische Jugendsozialarbeit (BAG EJSA) hat nun ein Konzept entwickelt, welches es Jugendlichen aus ihren Einrichtungen ermöglichen soll, an einem Freiwilligendienst teilzunehmen. Um die jungen Menschen dabei optimal zu unterstützen, werden erwachsene Freiwillige, die einen Bundesfreiwilligendienst ableisten, als ihre MentorInnen agieren und sie direkt am Einsatzort begleiten und bestärken: Jugendliche und MentorInnen sind „FiT - Freiwillig im Team“. Der vorliegende Beitrag stellt die neue Projektidee vor und zeigt Wege auf, wie junge benachteiligte Menschen für einen Freiwilligendienst erreicht werden können.

Schlagwörter: Benachteiligte junge Menschen; Freiwilligendienste; MentorInnen; Bundesfreiwilligendienst; Jugendfreiwilligendienst; Freiwilliges Soziales Jahr; Jugendsozialarbeit; Projektbeschreibung 


\section{Einführung und Ausgangslage}

Dieser Beitrag beschäftigt sich mit der Frage, warum benachteiligte junge Menschen bisher kaum in Freiwilligendiensten in Deutschland vertreten sind und stellt ein neues Projekt vor, in dem gezielt junge benachteiligte Menschen aus Einrichtungen der Jugendsozialarbeit ermutigt werden, ein Freiwilliges Soziales Jahr (FSJ) zu absolvieren. Im ersten Teil des Beitrags werden das Arbeitsfeld der Jugendsozialarbeit und der Bezug zu Freiwilligendiensten beleuchtet. Im zweiten Teil werden das Konzept und die Besonderheit des Projektes „FiT - Freiwillig im Team“ der Bundesarbeitsgemeinschaft Evangelische Jugendsozialarbeit e.V. (BAG EJSA) vorgestellt. Durch das Zusammenbringen von erwachsenen Freiwilligen, die gleichzeitig als MentorInnen für junge Menschen agieren, sollen Freiwilligendienste als Bildungs- und Beteiligungsmöglichkeit für bislang ausgeschlossene junge Menschen geöffnet werden.

Studien, wie der regelmäßig vom Bundesfamilienministerium durchgeführte Freiwilligensurvey (BMFSFJ 2010) zeigen, dass Freiwilligendienste immer noch überwiegend von jungen Menschen geleistet werden - insbesondere von jungen Frauen - die einen Abschluss der Mittleren Reife oder ein Abitur vorweisen können. Jugendliche mit niedrigem Bildungsabschluss oder Migrationshintergrund sind in den klassischen Vollzeitfreiwilligendiensten unterrepräsentiert (Engels, Leucht, Machalowski 2008: 131 ff.). Obwohl bereits seit Beginn der Evaluierung der Freiwilligendienste das Dilemma zwischen Bildungsstatus und freiwilligem Engagement in der Politik und bei den Trägern bekannt ist, scheiterten bisher die meisten Versuche, diese Gruppe in den Freiwilligendienst zu integrieren. Die Gründe hierfür sind immer noch nicht eindeutig ausgemacht und die Erklärungsversuche sind unterschiedlich. Ein häufig angegebener Grund liegt darin, dass Einsatzstellen in der Regel ältere und erfahrenere Jugendliche mit höherer Schulqualifikation bevorzugen und davor zurückschrecken, benachteiligte Jugendliche als Freiwillige aufzunehmen, da sie den Mehraufwand für die Betreuung der jungen Menschen scheuen bzw. nicht auffangen können. Es wird vorausgesetzt, dass die Freiwilligen „reibungslos funktionieren“.

Fakt ist, dass die Zugangshürden zu einem Freiwilligen Sozialen Jahr oder dem Bundesfreiwilligendienst für benachteiligte Jugendliche immer noch enorm hoch, und ihre Chancen einen Einsatzplatz zu bekommen eher gering sind. Hier will die BAG EJSA - als erfahrene Akteurin auf dem Gebiet 
Jugendsozialarbeit - mit ihrem neuen Konzept ansetzen und jungen Benachteiligten den Zugang zu einem Freiwilligendienst erleichtern, indem sie gleichzeitig eine zusätzliche Unterstützung durch MentorInnen schafft.

\section{Einrichtungen der Jugendsozialarbeit als Träger für Freiwilligendienste?}

\subsection{Kurzer Einblick in die Jugendsozialarbeit}

Jugendsozialarbeit ist kein Phänomen der aktuellen Zeit. Bereits in der Weimarer Republik existierten Formen der Unterstützung von beruflich ausgegrenzten und von Ausgrenzung bedrohten jungen Menschen. In der Nachkriegszeit wurde ein erstes, umfangreicheres System von Unterstützungsleistungen und Hilfeangeboten für benachteiligte junge Menschen installiert (Galuske 2005: 885 f.). Durch das Inkrafttreten des Kinder- und Jugendhilfegesetzes (KJHG) ${ }^{1}$ im Jahr 1991 und mit der Festschreibung des Begriffs Jugendsozialarbeit im §13 KJHG im Rechtsterminus erhielt die Jugendsozialarbeit wachsende Bedeutung (Galuske 2005: 886).

Allerdings gibt es bisher keine trennscharfe Definition des Begriffs ,Jugendsozialarbeit“. Sie ist weder deckungsgleich mit Sozialarbeit mit Jugendlichen noch mit Jugendberufshilfe: „Unter Jugendsozialarbeit lassen sich jene Maßnahmen und Angebote der Jugendhilfe zusammenfassen, die sich vorrangig und mittelbar der beruflichen und sozialen Integration von sogenannten benachteiligten bzw. individuell beeinträchtigten Jugendlichen und jungen Erwachsenen am Übergang von der Schule ins Erwerbssystem widmen“" (Galuske 2005: 886).

Nach dem SGB VIII soll die Jugendsozialarbeit ihre Angebote an alle sozial Benachteiligten und Beeinträchtigten richten, die am Übergang von Schule zu Beruf besonderer pädagogischer Betreuung bedürfen (Galuske 2005: 887). Sie hat daher den Fokus auf junge (sozial) benachteiligte Menschen bei deren Zugang zu beruflicher Qualifikation. Unter bildungs- und sozial benachteiligten Jugendlichen werden jene zusammengefasst, deren Subjektausstattung eine reibungslose Integration in den Ar-

\footnotetext{
${ }^{1}$ Sozialgesetzbuch (SGB) VIII
} 
beitsmarkt verhindert (Galuske 2005: 888). Die wesentliche Aufgabe der Jugendsozialarbeit ist demnach, die jungen Menschen dabei zu unterstützen, sich zu verantwortlichen, handlungsfähigen und handlungsbereiten Persönlichkeiten zu entwickeln. Sie leistet einen wichtigen Beitrag zur Überwindung und Verhinderung von jeglicher Benachteiligung und bekämpft gleichzeitig diskriminierende Einstellungen und Werte.

\subsection{Die Bundesarbeitsgemeinschaft Evangelische Jugendsozialarbeit}

Die Bundesarbeitsgemeinschaft Evangelische Jugendsozialarbeit e.V. (BAG EJSA) setzt sich als bundesweit tätige Fachorganisation der Jugendsozialarbeit und als Fachverband der Diakonie Deutschlands für die Belange junger Menschen ein, die besondere Unterstützung brauchen, um an unserer Gesellschaft teilhaben zu können. Sie ist diakonisch im Sinne von Dienst am Nächsten und an der Gesellschaft tätig. Dieses beinhaltet alltäglich praktizierte Nächstenliebe und drückt sich in dem aktiven Einsatz insbesondere für Schwache aus. Die BAG EJSA setzt sich dafür ein, dass jeder Mensch in Würde leben kann. Dabei geht sie von der Einzigartigkeit eines jeden Menschen aus. Ausgehend vom christlichen Menschenbild will sie mit ihren Angeboten eine Verbesserung von Teilhabe- und Entwicklungschancen für junge Menschen, die benachteiligt oder von Benachteiligung bedroht sind, erreichen sowie eine Verbesserung der Bildungschancen, die Förderung der außerschulischen Bildung und die Vernetzung der Bildungsorte (Familie Schule - Jugendsozialarbeit).

Aus diesem Selbstverständnis heraus leitet die BAG EJSA ihr Engagement für die Öffnung und Ermöglichung von Freiwilligendiensten für benachteiligte junge Menschen ab. Freiwilligendienste als ein entscheidender Schlüssel für gesellschaftliche Beteiligung und individuelle Bildung und Befähigung dürfen nicht länger jungen Menschen mit schwierigeren Startbedingungen verschlossen bleiben.

Im Folgenden Abschnitt wird daher zunächst dieser Begründungszusammenhang dargelegt und daraus die Entwicklung des Konzeptes für einen begleiteten Freiwilligendienst abgeleitet.

\subsection{Die Bedeutung von Freiwilligendiensten für junge Menschen}

Für eine aktive Zivilgesellschaft, an der sich alle gesellschaftlichen Gruppen beteiligen, haben Freiwilligendienste als geregelte Form bürgerschaftlichen 
Engagements einen wichtigen Stellenwert. Durch den Freiwilligendienst können die jungen Menschen ihre eigenen Fähigkeiten entdecken, nutzen und wertschätzen. Das Freiwillige Soziale Jahr - und seit dem 1. Juli 2011 auch der neu eingerichtete Bundesfreiwilligendienst - bieten den jungen Menschen die Möglichkeit neue Wissens-, Erfahrungs- und Sinnfelder zu erschließen und soziale Verantwortung für andere Menschen und das Gemeinwesen zu übernehmen (Liebig 2009: 12). Dieser Raum für persönliches, soziales und gesellschaftsbezogenes Lernen unterscheidet sich klar vom Lernen im Rahmen des formalen Bildungs- und Ausbildungssystems (EJSA 2008: 13).

Junge Menschen sind immer häufiger bis weit in das dritte Lebensjahrzehnt in der Rolle des/der SchülerIn bzw. des/der StudentIn. Dies bedeutet oftmals, dass sie unter sich bleiben (Entmischung der Generationen). Außerhalb des Schul- und Bildungssystems befinden sie sich jedoch in anderen Situationen, in welchen sie Erfahrungen und Kompetenzen sammeln sollen, die sie für ihre biographische Zukunft für entscheidend halten. Galuske bemerkt, dass die Lebensphase „Jugend“ in modernen Industriegesellschaften im Wesentlichen als Erprobung konzeptualisiert und funktionalisiert ist und eine Vorbereitung, Entwicklung und Einübung von akzeptablen Rollen, Orientierungsmustern, funktionalen und extrafunktionalen Qualifikationen für die spätere Erwachsenenexistenz zu sein scheint (Galuske 2005: 885).

Besonders jungen Menschen mit schlechteren Bildungs- und Startchancen gelingt es nur schwer, unter diesen Bedingungen eine Identitätsbildung zu leisten. Sie sind faktisch den Anforderungen der Umwelt nur mit professioneller Unterstützung, z. B. durch Jugendsozialarbeit, gewachsen. Insgesamt wird deutlich, dass benachteiligte junge Menschen eine Gesellschaft vorfinden, in der sie kaum Chancen für eine eigenständige und sinnstiftende Identitätsfindung und Teilhabe am Erwerbsleben haben (BMFSFJ 2013: 40 ff.). Insofern ist Jugendsozialarbeit aufgefordert, Angebote und Lösungen zu entwickeln, die auf die Lebenslagen des Einzelnen zugeschnitten sind. Freiwilligendienste sind so angelegt, dass sie die Identitätsbildung junger Menschen fördern können und diese Rollen im Erwerbssystem übernehmen und einüben können (Galuske 2005: 885).

Für benachteiligte junge Menschen ist die Teilnahme an einem Freiwilligendienst ein großer Gewinn für ihre berufliche Zukunft. Der Lernort Freiwilligendienst ermöglicht sozial Benachteiligten, solchen mit besonderem För- 
derbedarf oder bereits in den klassischen Bildungseinrichtungen Gescheiterten eine neue Art des Lernens (Liebig 2009: 12). So kann dieser Weg auch zu formalisierten Bildungsabschlüssen führen ${ }^{2}$; definitiv ist er jedoch ein hoher Wert für die individuelle Bildungsbiographie der jungen Menschen und kann ihre Chancen auf dem Arbeitsmarkt deutlich erhöhen (Liebig 2009: 64). „Gerade für Jugendliche, deren Lebenslauf sich naturgemäß nicht stark von gleichaltrigen Mitbewerbern unterscheidet, kann eine nachgewiesene Verantwortungsübernahme der ausschlaggebende Punkt für eine Einstellung sein“ (Liebig 2009: 64).

\subsection{Neue Rahmenbedingungen für bisher unerreichte Zielgruppen schaffen}

Um benachteiligten Jugendlichen einen Einsatz im Freiwilligendienst zu ermöglichen, sind entsprechende Rahmenbedingungen notwendig. Die Erfahrungen aus vorangegangenen Projekten der BAG EJSA und anderer Träger zeigen, dass sie eine zuverlässige, dauerhafte und kompetente Begleitung, Betreuung und Unterstützung (z. B. in Form einer Fachkraft mit pädagogischer Ausbildung, BildungspartnerIn/MentorIn) benötigen, die sie auch bei Fragen über den Freiwilligendienst hinaus konsultieren können. Weiterhin müssen die Anforderungen des Freiwilligendienstes neben den individuellen Fähigkeiten und Kenntnissen auch verstärkt an die Lebenslagen und Bedürfnisse der benachteiligten Freiwilligen angepasst sein. „Der ideale Freiwilligendienst für benachteiligte Jugendliche wird in lokale bzw. regionale Strukturen eingebunden sein, hält - zumindest zu Beginn des Dienstes ein Ensemble einfacherer Hilfstätigkeiten bereit, offeriert eine individuell abgestimmte fachliche Anleitung, bietet ein lückenloses Netz individueller Betreuung und verfügt über Strukturen, in denen Fehler toleriert werden können“ (Liebig 2009: 81). Bevor die Jugendlichen ihren Dienst beginnen, ist es daher ratsam, sie in die Strukturen der Einsatzstelle einzuführen, z. B. durch (einen) Hospitationstag(e). Die Aufgabe der Jugendsozialarbeit, eine Brücke zur Arbeitswelt für benachteiligte Jugendliche zu entwickeln und $\mathrm{zu}$ fördern und gleichzeitig nicht (nur) Schonraum für ihre AdressatInnen zu

2 Das Diakonische Werk Württemberg bietet für junge Menschen mit Hauptschulabschluss zwischen 18 und 26 Jahren das FSJ plus an. Innerhalb von zwei Jahren nehmen die Teilnehmenden an einem FSJ teil und erwerben den Realschulabschluss. 
sein, sondern vor allem ein Entwicklungsraum, in welchem junge Menschen mit dem Ziel gefördert werden, einen angemessenen Platz im Erwerbsleben zu finden, ist von besonderer Relevanz (Galuske 2005: 892). Freiwilligendienstprojekte in Zusammenarbeit mit Trägern der Jugendsozialarbeit sind daher nicht bloß eine weitere pädagogisch betreute Warteschleife oder ein Ersatz für eine Ausbildungsmaßnahme, sondern eine wichtige Erfahrung für (einige) benachteiligte junge Menschen über die Maßnahmen der Berufsorientierung und der beruflichen Ausbildung oder Qualifizierung hinaus, die ihre Chancen sichern helfen, ihnen eine Perspektive eröffnen und ihrem Leben einen Rahmen geben (Galuske 2005: 892). Sie bieten eine besondere Chance (bereits vorhandenen) Stigmatisierungsprozessen entgegenzusteuern, da sie eben nicht eine weitere Maßnahme der Jugendsozialarbeit sind und zweitens es sich um ein freiwillig wahrgenommenes Angebot handelt. Die jungen Menschen können daher ohne Druck soziale Kompetenzen erlernen und sich im Erwerbsleben ausprobieren.

Freiwilligendienste und Jugendsozialarbeit ergänzen einander und können gezielt genutzt werden, um Erfahrungen zu sammeln, frei von Stigmatisierungen und Leistungsdruck. Grundlage dafür sind Konzepte, die sich an den Voraussetzungen der jungen Menschen orientieren.

\section{Das Projekt Freiwillig im Team - „FiT“}

Aufgrund der oben genannten Bedeutung von Freiwilligendiensten für alle jungen Menschen und den bisher fehlenden nötigen Rahmenbedingungen für die Zielgruppe der benachteiligten Jugendlichen, hat die BAG EJSA das Konzept des Projektes „FiT“ entwickelt. In diesem Teil des Beitrags wird nun die Projektkonzeption vorgestellt sowie von ersten Erfahrungen berichtet.

\subsection{Projektidee}

Die Zielgruppe des Projektes „FiT - Freiwillig im Team“ sind (besonders) benachteiligte junge Menschen und Jugendliche mit besonderem Förderbedarf zwischen 18 und 25 Jahren, die beispielsweise:

- $\quad$ einen Haupt- oder Sonderschulabschluss oder keinen Abschluss vorweisen können, 
- $\quad$ die Schule oder eine Ausbildung abgebrochen haben oder SchulverweigerInnen sind,

- $\quad$ ohne Ausbildung oder Arbeit sind oder über längere Zeit keinen Ausbildungsplatz gefunden haben und daher seit längerem arbeitslos sind,

- $\quad$ mehrere Maßnahmen der Arbeitsagentur oder verschiedener Bildungseinrichtungen erfolglos durchlaufen haben,

- aus bildungsfernen Familien ohne Unterstützung von zuhause stammen,

- $\quad$ schlechte Deutschkenntnisse vorweisen,

- $\quad$ einen Migrationshintergrund oder Flüchtlingsstatus und eine weitere Benachteiligung haben.

Sie sollen ermutigt werden, sich für ein Freiwilliges Soziales $\mathrm{Jahr}^{3}$ als Vollzeitfreiwilligendienst in einer Einsatzstelle zu bewerben. Dieser Gruppe wird durch das Projekt ein Format angeboten, welches es ihnen ermöglicht, einen begleiteten Freiwilligendienst zu absolvieren. Dazu ist für das Projekt eine zweite Zielgruppe von besonderer Bedeutung und Wichtigkeit: Die Mentorinnen und Mentoren.

Für eine erfolgreiche Projektumsetzung wird zusätzlich in jeder teilnehmenden Einsatzstelle einE (pädagogisch) erfahreneR ältereR FreiwilligeR (MentorIn) im Rahmen des Bundesfreiwilligendienst eingesetzt. Da der Bundesfreiwilligendienst Menschen jeden Alters zugänglich ist, setzt die BAG EJSA die MentorInnen im Rahmen des BFD ein. Beide Zielgruppen - die jungen Menschen und die MentorInnen - absolvieren demnach gemeinsam einen Freiwilligendienst. Die MentorInnen haben die Aufgabe, gemeinsam mit den Einsatzstellen, die jungen Freiwilligen in ihren Angelegenheiten zu unterstützen und zu beraten sowie als AnsprechpartnerInnen bei Fragen oder Schwierigkeiten zur Verfügung zu stehen. Sie übernehmen die wichtige Aufgabe der pädagogischen Betreuung und Anleitung der Jugendlichen vor Ort und entlasten gleichzeitig durch ihre Arbeit die Fachkräfte in der Ein-

3 Aufgrund der Kontingentierung der verfügbaren Platzzahlen im Bundesfreiwilligendienst besetzt die BAG EJSA ihre FSJ Plätze mit den jungen benachteiligten Menschen. Die vorhandenen und altersoffenen Bundesfreiwilligendienstplätze werden für die Besetzung mit den MentorInnen benötigt. 
satzstelle. Der Mehraufwand für die Einsatzstellen wird somit erheblich reduziert und der Anreiz zur Einstellung eines benachteiligten jungen Menschen im Freiwilligendienst wird erhöht. Durch den Einsatz der MentorInnen können die bisher wenig oder gar nicht erreichten jungen Menschen in das System der Freiwilligendienste eingebunden werden. Für Jugendliche aus bildungsarmen oder benachteiligten Lebensräumen wird der Freiwilligendienst somit zugänglicher und attraktiver gemacht.

\subsection{Umsetzung der Projektkonzeption}

Für die Umsetzung des Projektes werden gezielt Einsatzstellen aus unterschiedlichen gemeinnützigen Bereichen gesucht. Dadurch soll gewährleistet werden, dass den jungen Menschen verschiedene Tätigkeitsbereiche (wie z. B. in den Bereichen Altenpflege, Haustechnik oder Hauswirtschaft) und Einsatzorte $^{4}$ angeboten werden können. Bei der Auswahl neuer Einsatzstellen achtet die BAG EJSA neben der Vielfältigkeit der Dienststätten auch besonders auf die Möglichkeit eines Anschlusses für die jungen Freiwilligen. Die jungen Menschen sollen Erfahrungen in Arbeitsfeldern sammeln, die diese später auch als Berufswunsch weiter verfolgen können. Tätigkeitsbereiche, die in der anschließenden Ausbildung ein hohen Schulabschluss oder gar ein Studienabschluss voraussetzen und dadurch für die Teilnehmenden am Projekt unerreichbar erscheinen, werden nicht in das Projekt aufgenommen.

Die erwachsenen MentorInnen, die mit den jungen Freiwilligen ihren Dienst beginnen, werden zu Beginn durch die anleitende Fachkraft in der Einsatzstelle in die wichtigen und relevanten Alltagsgeschäfte eingearbeitet. Anschließend können sie die jungen Freiwilligen in ihren Diensten optimal begleiten und sie in ihrer Zukunfts- und Berufsplanung unterstützen. Je nach Bereich arbeiten die MentorInnen in der Einsatzstelle auch normal mit. Neben der Begleitung durch die MentorInnen in der Einsatzstelle, werden die jungen Menschen vor Ort von einer Fachkraft mit angeleitet.

$\mathrm{Zu}$ den Hauptaufgaben der BAG EJSA als Träger gehören neben der Akquise neuer und geeigneter Einsatzstellen vor allem die bedarfserhöhte pädago-

${ }^{4}$ Das Projekt FiT wird zurzeit nur im Raum Baden-Württemberg angeboten. Eine deutschlandweite Ausweitung der Standorte ist in Planung. 
gische Begleitung und Beratung aller Freiwilligen (FSJ und BFD) sowie die Organisation, Verwaltung und Evaluation des gesamten Projektes. Gemeinsam mit den kooperierenden Einsatzstellen und unter Einbeziehung der Freiwilligen, werden die Seminare durch Unterstützung von fachlich erfahrenen und ausgebildeten ReferentInnen, mit speziell auf die Zielgruppen abgestimmten Themen organisiert. Diese dienen als Fortbildungsmaßnahme, Erfahrungsaustausch und Kompetenzerwerb für die Freiwilligen. Zusätzlich übernimmt die BAG EJSA als Träger die individuelle Begleitung und Betreuung aller Freiwilligen im Projekt und bietet dadurch die Möglichkeit, individuell und differenziert auf die Wünsche, Bedürfnisse und Problemlagen der Freiwilligen einzugehen. Die individuelle Begleitung erfolgt auf unterschiedlichen Wegen: durch Einzelgespräche vor Ort in den Einsatzstellen, während der Seminare sowie durch Kontakte über Telefon und Internet.

\subsection{Erste Erfahrungen und Ausblick}

Erste Erfahrungen zeigen, dass Einsatzstellen des FSJ Interesse an dem Konzept und dem Projekt äußern. Hervorzuheben ist hier die Motivation der sozialen Einrichtungen - in Zeiten des Fachkräfte- und Nachwuchsmangels bedingt durch den demografischen Wandel - sich neuen Zielgruppen zu öffnen und andere Wege zu gehen, offene Ausbildungsplätze zu besetzen. Das Konzept des Freiwilligentandems stößt auch deshalb auf positive Resonanz, da eine umfassende Betreuung und Beratung durch den Träger gewährleistet wird und die MentorInnen zusätzliche Belastungen für die Fachkräfte in den Einrichtungen abfangen und gleichzeitig personelle Unterstützung für alltäglich anfallende Arbeiten sind. Freiwillige im FSJ und BFD übernehmen in den Einrichtungen häufig Aufgaben, die im Berufsalltag der Fachkräfte zu kurz kommen und für die in der Regel kaum Zeit bleibt, bspw. ausführliche Unterhaltungen oder Spaziergänge mit BewohnerInnen eines Altenheimes.

Zurzeit bietet die BAG EJSA zehn benachteiligten jungen Menschen und zehn MentorInnen (also insgesamt zehn Tandems) einen Freiwilligenplatz an. Eine Ausweitung der Einsatzstellen und der Platzzahlen, so dass insgesamt mehr Tandems eingesetzt werden können, ist ein wesentliches Ziel des Projektes, an dessen Umsetzung kontinuierlich gearbeitet wird.

Das Projekt ist vorerst auf drei Freiwilligenjahrgänge - bis August 2015 angesetzt. Während dieser Zeit wird die Projektkonzeption erprobt und - wo 
nötig - weiterentwickelt. Ab dem Freiwilligenjahrgang 2015/2016 soll das Projekt soweit evaluiert sein, dass eine dauerhafte Umsetzung des Konzeptes eingeführt werden kann. Aktuelle Informationen und Fortschritte werden regelmäßig auf der Projekthomepage www.freiwillig-im-team.de veröffentlicht und können dort nachgelesen werden.

Noch kann die BAG EJSA nicht auf umfangreiche Erfahrungen zurückgreifen, da sich das Projekt FiT in der Anfangsphase befindet. Wir erhoffen uns von dem Projekt die oben beschriebenen Wirkungen und setzen uns mit ganzer Kraft für die erfolgreiche Umsetzung ein.

\section{Literaturverzeichnis}

BMFSFJ (2013): 14. Kinder- und Jugendbericht: Bericht über die Lebenssituation junger Menschen und die Leistungen der Kinder- und Jugendhilfe in Deutschland, Rostock.

BMFSFJ (2010): Hauptbericht des Freiwilligensurveys 2009. Zivilgesellschaft, soziales Kapital und freiwilliges Engagement in Deutschland 1999 - 2004 - 2009, München.

Bundesarbeitsgemeinschaft Evangelische Jugendsozialarbeit (2011): Lebensmittel Bildung. Evangelische Jugendsozialarbeit für Befähigung und Teilhabe, Stuttgart.

Bundesarbeitsgemeinschaft Evangelische Jugendsozialarbeit (2008): Ziele, Aufgaben, Struktur Fachkonzept, Stuttgart.

Engels, Dietrich, Martina Leucht und Gerhard Machalowski (2008): Evaluation des freiwilligen sozialen Jahres und des freiwilligen ökologischen Jahres, Wiesbaden.

Galuske, Michael (2005): Jugendsozialarbeit und Jugendberufshilfe, in: Handbuch Sozialarbeit Sozialpädagogik, hrsg. von Hans-Uwe Otto und Hans Thiersch, München, S.885-893.

Liebig, Reinhard (2009): Freiwilligendienste als außerschulische Bildungsinstitution für benachteiligte junge Menschen, Machbarkeitsstudie in Auftrag gegeben und herausgegeben vom Bundesministerium für Familie, Senioren und Jugend, Wiesbaden. 


\begin{abstract}
:
Maike Herrmann

„FiT” - Voluntary Team Work

Disadvantaged young people in voluntary social services

Young people with low educational achievement or socially disadvantaged young persons are still underrepresented in voluntary social services in Germany. Such services so far have been offered rarely to young disadvantaged adolescents since appropriate structures and frameworks are often missing. The Bundesarbeitsgemeinschaft Evangelische Jugendsozialarbeit (Association of protestant social youth work) now developed a new concept for disadvantaged young people to participate in voluntary social services in Germany. For an ideal support the young volunteers are accompanied by adults, so called mentors, who encourage the young people during their social service: Young people and the mentors are FiT - Voluntary team work. This article pictured the new project idea and shows how to reach disadvantaged young people to get involved with voluntary social services.
\end{abstract}

Keywords: Disadvantaged young people; Federal Voluntary Service; Mentoring; voluntary social services; youth social work 